\title{
Conformation Changes, Complexation, and Phase Transition in Matrix-Assisted Laser Desorption
}

\author{
Mehrnoosh Sadeghi, Xiongwu Wu, and Akos Vertes* \\ Department of Chemistry, George Washington University, Washington, D.C. 20052
}

Received: September 13, 2000; In Final Form: January 4, 2001

\begin{abstract}
Using molecular dynamics simulations we have demonstrated that the guest species in matrix-assisted laser desorption exhibits significantly different conformations in the gas phase, on the crystal surface, and embedded into the matrix. Our model fully accounted for the internal structure of matrix and guest molecules; thus, we were able to follow the details of conformational changes in the guest molecule within the crystal and throughout the desorption. During the embedding process, simulated by sinapinic acid crystal growth around the substance $\mathrm{P}$ guest species, changes in the guest species conformation were investigated. While the guest species placed on the surface of the host crystal exhibited two hydrogen bonds on the backbone and one turn, the conformation of the embedded species contained three hydrogen bonds and two turns. Independent of the burial depth, the conformation of the guest species within the crystal remained the same. After laser heating and during the desorption process, no preferred gas-phase conformation was observed. Investigation of the radius-of-gyration of the guest species originating from different layers of the crystal revealed that, upon desorption, the guest species did not sustain a stable conformation in the gas phase. Liftoff velocities of the guest species embedded in various layers of the host crystal and on the surface were similar to the liftoff velocities of the matrix molecules around them. This is an indication of the entrainment of guest species by the matrix plume. Investigation of the energy histories of the matrix and guest species showed lower internal and kinetic temperatures for the latter, which could explain the absence of fragmentation in MALDI. Results also showed that maximum energy transfer occurred during the phase transition of the host crystal at which time the guest species were released from the crystal. Examining the noncovalent complex of the guest species and sinapinic acid anions showed that the complex remained intact throughout the desorption process. A similar phenomenon is often reflected in MALDI mass spectra, in the form of guest-matrix adduct peaks.
\end{abstract}

\section{Introduction}

During the past decade, matrix-assisted laser desorption ionization (MALDI) has proven to be one of the most efficient sources of intact ions for large biopolymers. In MALDI, the difficulty of volatilizing large molecules is overcome by laser irradiation of a cocrystallized sample of the molecule of interest and a matrix. The presence of the matrix that exhibits low phase transition temperature and strong absorption at the excitation wavelength spares the guest species from decomposition upon laser energy deposition. As a result, molecules with molecular masses up to $1000 \mathrm{kDa}$ have been detected by MALDI mass spectrometry. ${ }^{1,2}$

In addition to the factors that directly influence the quality of a MALDI mass spectrum (laser irradiance and wavelength, ion velocity distributions, sample preparation, etc.), evidence is accumulating regarding the influence of parameters of a less direct nature on the MALDI phenomenon: for example, the effect of the secondary structure (conformation) of protein guest molecules on the ion yield, ${ }^{3,4}$ the effect of the ambient pressure on the plume dynamics (atmospheric pressure MALDI), ${ }^{5}$ or the effect of noncovalent complex formation on the MALDI process. ${ }^{6}$ Some of the reported data show that peptides with stable $\alpha$-helical and $\beta$-sheet structures exhibit lower signal intensities than peptides with disturbed secondary structures. ${ }^{3}$

* To whom correspondence should be addressed. Phone: (202) 994 2717. Fax: (202) 994-5873. E-mail: vertes@gwu.edu.

10.1021/jp0032715 CCC: \$20.00
These results point to the importance of guest species conformation and to the need for investigating conformational changes during the MALDI process. Some of the observations are also driving new applications, such as studying the gas-phase conformation of macromolecules using MALDI in combination with ion mobility spectrometry ${ }^{7-9}$ or extending MALDI ionization to an atmospheric environment. ${ }^{5,10}$ These applications reinforce the need for a realistic description of MALDI including not only the desorption event but also the embedding process during sample preparation.

For proteins, five potentially different macromolecular conformations play a role in the MALDI process. Initially there is the native protein conformation in the aqueous solution. Upon mixing the sample with the concentrated acidic matrix solution, the $\mathrm{pH}$ drops significantly (saturated solutions of common matrices exhibit $\mathrm{pHs}$ in the $1.7-3.1$ range $^{6}$ ). This $\mathrm{pH}$ drop leads to the protonation of the amino terminus and the basic residues (Arg, Lys, His) and results in the stretching of the polypeptide chain. During the cocrystallization of the matrix and guest molecules, the protonated sites on the guest species are partially neutralized by anions mostly from the dissociated matrix. Before embedding, this ion-associate assumes a specific conformation on the surface of the growing matrix crystal. Upon embedding, the protein may assume yet another conformation due to the interactions with the surrounding matrix molecules. Finally, when laser heating liberates the protein from the host crystal, another conformation change is expected. The time scale of this 
conformation change is commensurate with the formation of the MALDI plume $(<100 \mathrm{~ns})$, whereas the time scales of the previous conformation changes are much longer (e.g., crystallization takes place in $10-100 \mathrm{~s}$ ). For certain proteins, conformational data are available for an aqueous environment at different pHs. Gas-phase conformation can also be extracted from ion mobility measurements. ${ }^{7-9}$ No information is available on the conformation changes occurring during the guest-matrix cocrystallization and during the desorption process.

Due to the significance of substance P (SP) as a neuropeptide, a number of reports are available on the conformation of this molecule in various environments. ${ }^{1-16}$ Different studies have shown that SP has no preferred conformation in aqueous solutions. ${ }^{11,12,15}$ A mixed $\alpha$-helix/folded structure in methanol has been proposed with five hydrogen bonds determining the conformation of the molecule. ${ }^{12}$ Due to the participation of SP in membrane interactions, the structure of this molecule has been identified in the vicinity of cell membranes. SP is suggested to contain a nine-residue $\alpha$-helix at the $\mathrm{C}$-terminus and a random coil at the N-terminus in the lipid membrane ${ }^{13}$ or two type-I $\beta$-turns in a biphasic membrane mimic. ${ }^{16}$ In the solid state $(\mathrm{KBr}$ disk) SP is shown to exhibit a main random coil and/or $\alpha$-helical structure. In addition, there is evidence for a $\beta$-structure in this phase. ${ }^{14}$ Measured and calculated gas-phase collision crosssections are available for the singly and doubly charged forms of SP. While the ion mobility experiments show a more compact structure (smaller cross-section) for the doubly charged ions, a molecular dynamic study of these two charged states predicts identical cross-sections. ${ }^{17}$ This apparent contradiction is rationalized by self-solvation of the protonated basic sites (Arg, Lys) by carbonyl oxygens carrying partial negative charges in the different species. ${ }^{17}$

Details of the interaction between the guest and matrix molecules within the crystal are among the unexplored issues in MALDI. Due to the specific molecular environment in MALDI matrices, the conformation of the embedded biomolecules is expected to be influenced by the crystal structure of the matrix. The number of reports on the inclusion of the guest molecule within the matrix crystal and information pertaining to their interactions is limited. ${ }^{18-20}$ The first result of this kind was reported by Beavis and Bridson in 1993. ${ }^{18}$ The authors have shown, using X-ray crystallographic data, that the hydrophobic hydrogen bonded extended sheets of 3,5-dimethoxy-4-hydroxycinnamic acid (trans-sinapinic acid or SA) on the (103) plane are responsible for the inclusion of proteins and that the guest molecule exhibits an extended conformation within the crystal. More recent investigations of the crystal structure of 2,5dihydroxybenzoic acid and succinic acid matrices, however, show no such hydrophobic domains. ${ }^{19}$ Therefore, it is suggested that the incorporation of proteins into the matrix crystal can proceed through a variety of interactions. A study of cytochrome $C$ in different isomers of dihydroxybenzoic acid shows that while efficient guest incorporation into the matrix crystal leads to a superior MALDI signal, intimate surface contact between the two species is also sufficient. ${ }^{20}$

Noncovalent complexes play a significant role in the regulation of biological processes. There have been indications that certain complexes survive the sample preparation and laser desorption conditions in MALDI. ${ }^{6,21,22}$ The strongest noncovalent forces are based on Coulomb interactions between fully and/or partially ionized sites. For example, complex formation between peptides and oligonucleotides is facilitated by the presence of protonated basic amino acid residues in the peptide and negatively charged phosphate groups in the oligonucleotide. ${ }^{6}$
It is not known, however, what makes some of these complexes survive the MALDI process. Similarly, nothing is known about the dynamic behavior of these complexes in the MALDI environment. Clearly, the breakup of the complex can be the result of either the $\mathrm{pH}$ changes associated with the MALDI sample preparation or the extra energy acquired during the laser desorption process. The fate of this energy is also of interest: it is not clear how much is expended on raising the internal energy of the individual components and what fraction goes into loosening the complex itself.

Experimental investigation of the early dynamics of the desorption ( $<20 \mathrm{~ns}$ time scale) is just beginning to emerge. ${ }^{23,24}$ The phase transition necessary to release ions from the solid sample is shown to occur in less than $1 \mathrm{~ns}^{24}$ This is followed by the production of a dense plume ( $<10 \mathrm{~ns})$ with an abundance of clusters and a transition into a rarified plume $(<100 \mathrm{~ns})$ composed primarily of single molecules. To address the details of desorption in MALDI at the microscopic level, molecular dynamics (MD) simulations have been applied. ${ }^{25-33}$ The dynamics of desorption and hydrogen bonding was first described by Bencsura and Vertes in a hemispherical volume of nicotinic acid matrix seeded with leucine enkephalin guest species. Using selective excitation of the matrix molecules, the energy transfer between matrix and guest molecules was also investigated for the same system. ${ }^{25,26}$ The results supported the previously developed homogeneous bottleneck model that had rationalized the absence of fragmentation in MALDI. ${ }^{34,35}$ To expand the time and size scale in the MD studies of MALDI, Zhigilei and Garrison introduced the breathing sphere model. ${ }^{27,28,31-33}$ Their results indicated the presence of a fluence threshold separating layer-by-layer desorption from ablation, the formation of clusters in the plume, and the peculiarities of the velocity distributions for the two species. Due to the need to keep the calculations manageable, this model employed a single parameter (the radius of the breathing sphere) for the representation of the internal degrees of freedom. In a recent study, $\mathrm{Wu}$ et al. have reported simulations that accounted for most of the internal degrees of freedom in the nicotinic acid leucine enkephalin host-guest system. ${ }^{29}$ Liftoff velocities, guest conformation changes, and energy transfer between the matrix and guest molecules were reported for this system. To account for nonlinearities in the interaction potential, Dutkiewicz et al. have investigated laser heating of a system of small molecules. Utilizing the accurate interaction potentials available for oxygen molecules, melting was identified as the prerequisite for the efficient vibrational to translational energy transfer processes and for the catastrophic desorption from solid oxygen crystals. ${ }^{30}$

It has long been demonstrated that the nature of the matrix and guest molecules significantly influences the MALDI spectrum. For example, the choice of matrix has been shown to affect the fragmentation process by controlling the internal energy of the guest species. ${ }^{36}$ In addition, different guest molecules show different degrees of fragmentation upon laser irradiation (e.g., compared to proteins, oligonucleotides show higher propensity for fragmentation). Therefore, comparison of different matrices and guest molecules at the microscopic level are of interest for unveiling the structure-specific features of the desorption processes in MALDI.

In our previous studies, nicotinic acid-leucine enkephalin systems were selected to investigate the desorption phenomena in MALDI. In the present report, we have chosen a larger guest species (SP, $1347 \mathrm{Da}$ ) to allow for a better study of conformational changes during embedding and desorption. In addition, we used SA, one of the most common matrices in the analysis 
of proteins, as the matrix. Moreover, the kinetic and internal energies of the guest and matrix molecules are treated separately. Such a treatment gave us insight into the details of the energy transfer during desorption and release of the guest molecules from the host crystal. We also took a closer look at the matrix phase transition during the desorption process and the stability of noncovalent complexes between ionized matrix and guest molecules.

\section{Modeling Method}

Details of the MD calculations have been described elsewhere. ${ }^{29}$ Briefly, the $\mathrm{c} 24 \mathrm{~b} 2$ version of the MD code CHARMM (CHARMM Development Project, Harvard University, Cambridge, MA) was used for the simulations. ${ }^{37}$ The empirical potential energy function adopted in CHARMM to model macromolecular systems consists of the bond stretching, bending, improper dihedral, dihedral, van der Waals, and electrostatic terms. The original CHARMM database does not contain the interaction potential parameters for the matrix molecules (e.g., SA); thus, these had to be calculated and tested separately (see later). To reduce the complexity of the MD simulations, chemical transformations of the participating species were not allowed. The guest molecule, SP, and the host molecules, SA, were represented using a realistic description of their molecular structures. In this "all atom" representation, every atom in the system is explicitly included in the calculations. The atom numbering assignments in SA are shown below.

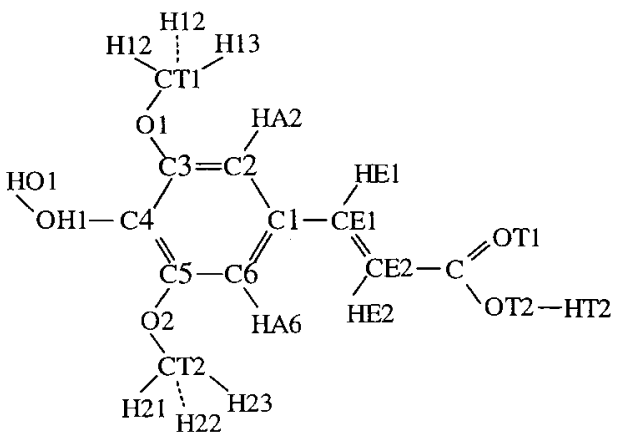

Partial charges of the atoms in SA were calculated using the GAMESS ab initio program for the fully optimized structure at the 6-31G* level (see values in Table 1). ${ }^{38}$ To build the proper interaction potential for SA, the partial charge values were incorporated into the CHARMM parameter file. Interaction parameters for SA were also estimated from similar compounds and groups in the CHARMM parameter file.

The initial crystal structure of SA was built on the basis of $\mathrm{X}$-ray diffraction data determined by Beavis and Bridson. ${ }^{18}$ The SA crystal is described as monoclinic with $P 2_{1} / n$ symmetry with $\mathrm{Z}=4$, with the lattice parameters $a=4.760 \AA, b=15.686 \AA$, $c=14.185 \AA$, and $\beta=90.30^{\circ}$ and with a calculated density of $1.406 \mathrm{~g} / \mathrm{cm}^{3}$. Characteristic to similar organic acids, the SA molecules in the crystal form carboxyl-bound dimers and arrange into hydrogen-bonded sheets. The $x-y$ dimensions of the modeled crystal region were $60.225 \times 47.058 \AA^{2}$, containing 11 sheets of 36 SA molecules (in each sheet) with the (103) plane aligned horizontally. To model a semiinfinite solid, twodimensional periodic boundary conditions were applied to this cell. The two bottom layers were anchored to represent the bulk of the crystal. To incorporate the interaction of the fixed layers with the layer of molecules free to move in the simulations, the number of the rigid layers was set so that their total thickness was less than the cutoff distance of the nonbonded interactions
TABLE 1: Atom Types and Partial Charges in the SA Matrix Molecule

\begin{tabular}{clcc}
\hline atom no. & atom & atom type $^{a}$ & partial charge $^{b}$ \\
\hline 1 & C1 & CA & -0.15 \\
2 & HA2 & HP & 0.19 \\
3 & C2 & CA & -0.26 \\
4 & H11 & HA & 0.10 \\
5 & H12 & HA & 0.04 \\
6 & H13 & HA & 0.04 \\
7 & CT1 & CT3 & 0.05 \\
8 & O1 & OS & -0.38 \\
9 & C3 & CA & 0.42 \\
10 & HO1 & H & 0.50 \\
11 & OH1 & OH1 & -0.63 \\
12 & C4 & CA & -0.04 \\
13 & H21 & HA & 0.05 \\
14 & H22 & HA & 0.11 \\
15 & H23 & HA & 0.05 \\
16 & CT2 & CT3 & 0.05 \\
17 & O2 & OS & -0.47 \\
18 & C5 & CA & 0.51 \\
19 & HA6 & HP & 0.26 \\
20 & C6 & CA & -0.43 \\
21 & HE1 & HA1 & 0.15 \\
22 & CE1 & CE1 & -0.04 \\
23 & HE2 & HA1 & 0.09 \\
24 & CE2 & CE1 & -0.13 \\
25 & C & CD & 0.64 \\
26 & OT1 & OB & -0.66 \\
27 & OT2 & OH1 & -0.58 \\
28 & HT2 & H & 0.52 \\
& & & \\
& & &
\end{tabular}

${ }^{a}$ These atom types are defined in the CHARMM residue topology file. ${ }^{b}$ Partial charge values were calculated by GAMESS at the $6-31 G^{*}$ level.

(13 $\AA$ ). The 2-D periodic boundary condition along the $x-y$ plane and the rigid layers at the bottom allowed evaporation of the system only at the top surface perpendicular to the $z$-axis.

Due to the acidic environment of MALDI matrices, the basic amino acid residues $\left(\operatorname{ArgH} H^{+}, \mathrm{p} K_{a}=8.0 ; \mathrm{LysH}^{+}, \mathrm{p} K_{a}=10.0\right.$; $\left.\mathrm{HisH}^{+}, \mathrm{p} K_{a}=6.5\right)$ and the amino terminus $\left(\mathrm{NH}_{3}{ }^{+}, \mathrm{p} K_{a}=8.0\right)$ of peptides and proteins are expected to be protonated. ${ }^{6}$ In saturated SA the $\mathrm{pH}=3.1$; thus, we chose the +3 charged ion of the naturally occurring undecapeptide, SP, as the guest species in these simulations:

\section{$\mathrm{NH}_{3}{ }^{+}$- $\mathrm{ArgH}{ }^{+}$-Pro-LysH ${ }^{+}$-Pro-Gln-Gln-Phe-Phe-Gly- \\ Leu-Met- $\mathrm{NH}_{2}$}

Potential energy parameters for the amino acid residues were taken from the CHARMM parameter file. Since the modeled $\mathrm{SP}^{3+}$ species carried three positive charges, to maintain charge neutrality it was surrounded by three $\mathrm{SA}^{-}$anions. The matrix anions were placed near to the positively charged groups in $\mathrm{SP}^{3+}$; i.e., the $\mathrm{COO}^{-}$of the $\mathrm{SA}^{-}$ions was placed in the vicinity of the protonated amino terminus, $-\mathrm{NH}_{3}{ }^{+}$, and the protonated $=\mathrm{NH}_{2}{ }^{+}$and $-\mathrm{NH}_{3}{ }^{+}$moieties in the side chains of $\mathrm{ArgH}^{+}$and $\mathrm{LysH}^{+}$, respectively. The system of $\mathrm{SP}^{3+}$ and $\mathrm{SA}^{-}$anions, $\left[\mathrm{SP}^{3+}\right.$ $+3 \mathrm{SA}^{-}$], was energy minimized for 100 steps using the adopted basis Newton-Raphson method.

In the calculations, the incorporation of the guest species into the matrix followed the likely mechanism of the natural embedding process during cocrystallization. The process was initiated by the adsorption of the peptide onto the SA crystal surface followed by layer-by-layer embedding due to rapid crystal growth. Four different host-guest systems were investigated in which the number of SA layers placed on top of the $\left[\mathrm{SP}^{3+}+3 \mathrm{SA}^{-}\right]$complex was varied to explore the effect of burial depth. 
In the initial adsorption simulation, the extended $\mathrm{SP}^{3+}$ and the three $\mathrm{SA}^{-}$anions $\left(\left[\mathrm{SP}^{3+}+3 \mathrm{SA}^{-}\right]\right)$were positioned on the top of the (103) surface of the SA crystal (Figure 1). The system was then equilibrated for $100 \mathrm{ps}$. To model crystal growth, layers seven to nine of the matrix structure were moved above the surface and the $\left[\mathrm{SP}^{3+}+3 \mathrm{SA}^{-}\right]$adsorbate. The SA molecules within a radius of $2.4 \AA$ around the guest species were deleted from the host crystal. The $\left[\mathrm{SP}^{3+}+3 \mathrm{SA}^{-}\right]$complex thus seated in the third layer extended into two layers. This process was repeated two more times, creating systems with the guest species in the sixth and ninth layers of the eleven-layer host system. In all model systems the two bottom layers of SA molecules were restricted in their motion to model the effect of the crystal bulk. Each of the four systems was equilibrated afterward for $100 \mathrm{ps}$ at $300 \mathrm{~K}$. These four systems served as starting points for the laser excitation simulations.

Selective coupling of the laser energy to the system was simulated by mainly heating the matrix molecules using the method introduced by Nose and Hoover. ${ }^{39,40}$ Two constant heat baths were coupled to the system, and the heat-transfer rates were controlled by the appropriate assignment of two parameters, i.e., the thermal inertial momentum and the velocity of the scale parameter. Adjusting these properties of the two heat baths allowed us to individually control the energy transfer to the guest and matrix molecules in a way that direct heating of the matrix molecules was achieved while the guest species were only heated through their interaction with the matrix molecules. Rapid heating of the matrix was carried out at a rate that represented the deposition of energy during the laser pulse.

The temperature of the heat bath assigned to the matrix molecules during desorption was $2000 \mathrm{~K}$ while the guest species were coupled to a $300 \mathrm{~K}$ bath. To set the matrix heat bath temperature, we experimented with values ranging from 1000 to $2500 \mathrm{~K}$. The selected value, $2000 \mathrm{~K}$, was chosen because lower temperatures did not lead to evaporation in the time frame of the simulation. The thermal inertial momentum which controlled the rate of temperature fluctuations of the two heat baths was set to maintain a one-way continuous heat flow during the desorption process. The energy flow rate between the baths and the subsystems was controlled by the velocity of the scale parameter, $\dot{s}$. To prevent the energy flow between the guest heat bath and the guest species, we set $\dot{s}_{\text {guest }}=0$. However, to allow gradual energy flow from the matrix heat bath to the matrix molecules $\dot{s}_{\mathrm{m}}=-0.001$ was selected. The typical time step for the simulations was $2 \mathrm{fs}$, and the coordinates and velocities were saved at $0.2 \mathrm{ps}$ intervals for later analysis.

Simulations were performed and analyzed on Indigo 2 (with R4400 processor) and Indy (with R4600 processor) workstations operating under IRIX 5.3 and IRIX 6.2, respectively (Silicon Graphics, Mountain View, CA) and on a Sun Ultra Enterprise 4000 system (Sun Microsystems, Mountain View, CA) operating under SunOS 5.5.1. System structures and trajectories were visualized and monitored by the SCARECROW (Centre for Scientific Computing, Espoo, Finland), ${ }^{41}$ the RasMol (Glaxo Wellcome R\&D, Hertfordshire, U.K.), ${ }^{42}$ and the MOLMOL (Institute of Molecular Biology and Biophysics, ETH, Zürich, Switzerland $)^{43}$ packages.

\section{Results and Discussion}

To validate the interaction potentials introduced for SA and the stability of our system, thermal and structural properties of the SA crystal were calculated. The most relevant thermal property governing the laser induced phase transition is the enthalpy of sublimation, $\Delta H_{\text {subl. }}$. Its value was obtained by subtracting the enthalpy of the host crystal in equilibrium configuration from the enthalpy of a system with one SA molecule transferred into the gas phase. At $300 \mathrm{~K}$, the enthalpy of sublimation for SA was calculated to be $130 \mathrm{~kJ} / \mathrm{mol}$. Although the experimental value for $\Delta H_{\text {subl }}$ of SA is not available, the calculated value is similar to $\Delta H_{\text {subl }}$ of other MALDI matrices such as nicotinic acid $(123.4 \mathrm{~kJ} / \mathrm{mol})$, succinic acid $(120.0 \mathrm{~kJ} /$ $\mathrm{mol})$, and cinnamic acid $(109.6 \mathrm{~kJ} / \mathrm{mol})$.

To further verify the SA interaction potential, we compared the equilibrated crystal structure to the experimental one. Radial distribution functions were calculated for certain atoms in the SA molecule. For example, for the CE1 atom we generated the radial distribution function. It exhibited peaks at $4.74,15.5$, and $14.1 \AA$ that compared well with $a=4.76 \AA, b=15.7 \AA$, and $c=14.2 \AA$ for the SA unit cell. After the verification process, the guest species were introduced into the system.

Matrix Crystal Growth and Embedding of the Guest Species. To follow the conformational changes of SP during the adsorption and embedding process, initially the guest species was deposited onto the surface of the matrix and the crystal was gradually grown around it. This procedure was designed to simulate cocrystallization during the sample preparation process.

Initially, previously equilibrated guest species were placed on the free (103) surface of the SA crystal and further equilibrated at $300 \mathrm{~K}$ for $100 \mathrm{ps}$. Figure 1 shows the side view (Figure 1a) and the top view (Figure 1b) of the equilibrated system. In the resulting conformation, the $\mathrm{SA}^{-}$anions maintained their position close to the protonated sites in the $\mathrm{SP}^{3+}$ species. Each $\mathrm{SA}^{-}$anion interacted with two of the three charged sites of $\mathrm{SP}^{3+}$ (the protonated side chains of Arg and Lys and the amino terminus) acting as a bridge. At residues $G \ln (5)$ and $\mathrm{Gln}(6)$ of $\mathrm{SP}^{3+}$ (Figure 2a), there was a turn to arch the peptide chain away from the crystal surface near the C-terminus. A hydrogen bond was observed on the backbone between the $\mathrm{O}$ in the peptide bond of Pro(4) and the $\mathrm{HN}$ in $\mathrm{Gln}(6)$ ( $2_{7}$ ribbon). A second hydrogen bond was observed between the two Phe $(8$ and 9) residues. The $\mathrm{SP}^{3+}$ exhibited one turn and up to nine hydrogen bonds between the side chains and peptide bonds on the backbone.

After moving three layers of SA molecules to the surface, the guest species became buried in the third layer of the host crystal. The position of the $\mathrm{SA}^{-}$ions relative to the guest species remained unchanged. However, upon the growth of the first crystal layer above the $\left[\mathrm{SP}^{3+}+3 \mathrm{SA}^{-}\right]$complex, the conformation of the guest species changed from its initial surface conformation to exhibit two turns and three hydrogen bonds on the backbone (Figure 2b). In addition to the turn at Pro(4) and $\mathrm{Gln}(6)$, a $\beta$-turn was observed between Phe(7) and Leu(10). A third hydrogen bond was also seen between the HN of the peptide bond in Gly(9) and the peptide bond O in Leu(10). Seven hydrogen bonds were observed between atoms in the side chains and the backbone. The $\left[\mathrm{SP}^{3+}+3 \mathrm{SA}^{-}\right]$complex was extended into two layers of matrix molecules. The matrix molecules surrounding the guest species were displaced from their initial crystal position and orientation.

Three additional matrix layers were then moved to the top of the second system, embedding the guest species into the sixth layer. The guest species within the $\left[\mathrm{SP}^{3+}+3 \mathrm{SA}^{-}\right]$complex maintained a conformation and hydrogen bond system similar to that in the third layer. By moving three more layers of SA molecules to the top of the third system, a final system with the guest species in the ninth layer was created. This placed the guest species right above the two fixed layers of matrix 


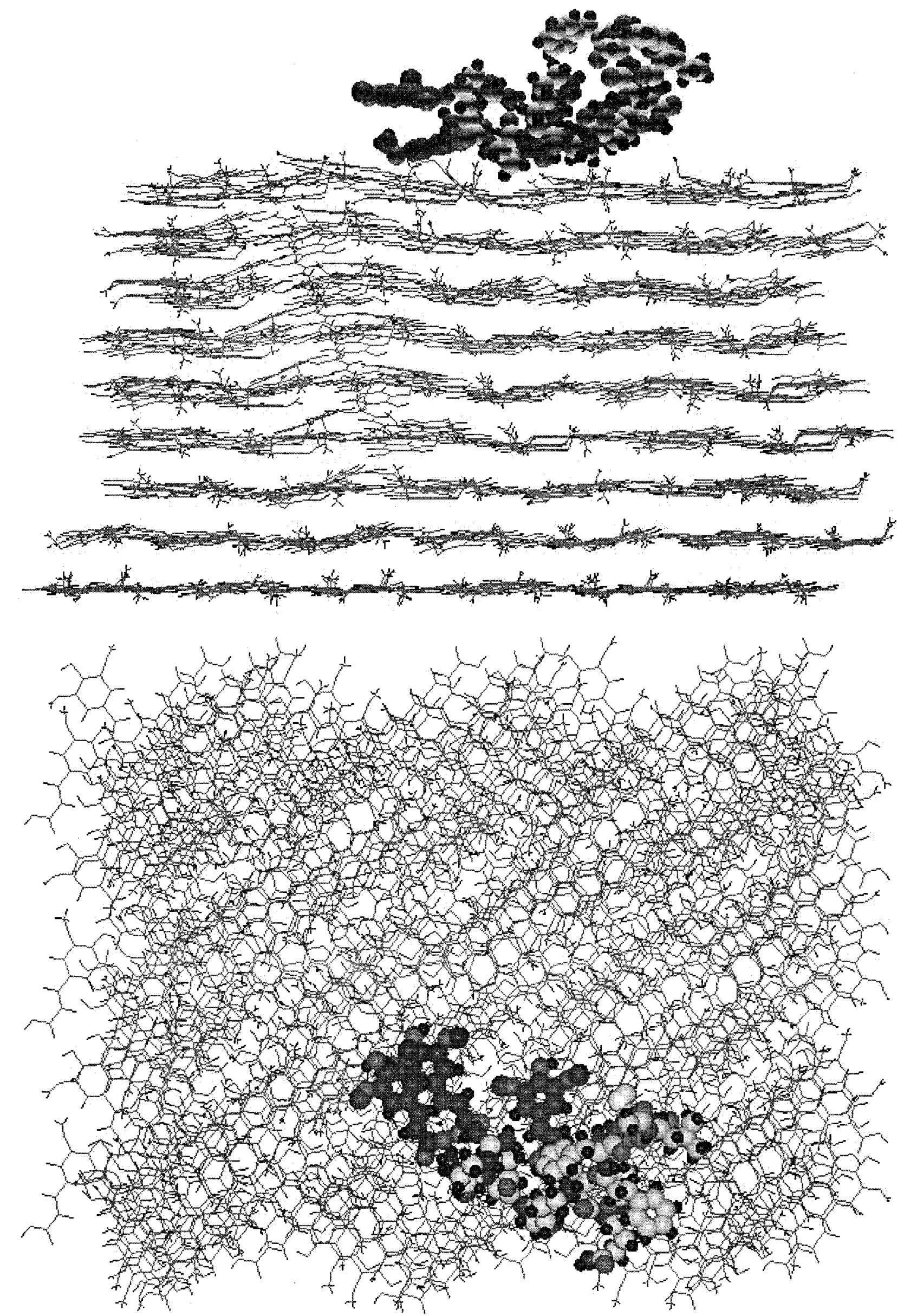

Figure 1. Equilibrated $\left[\mathrm{SP}^{3+}+3 \mathrm{SA}^{-}\right]$species on the (103) surface of SA crystal at $300 \mathrm{~K}$ : (a, top) side view and (b, bottom) top view. 

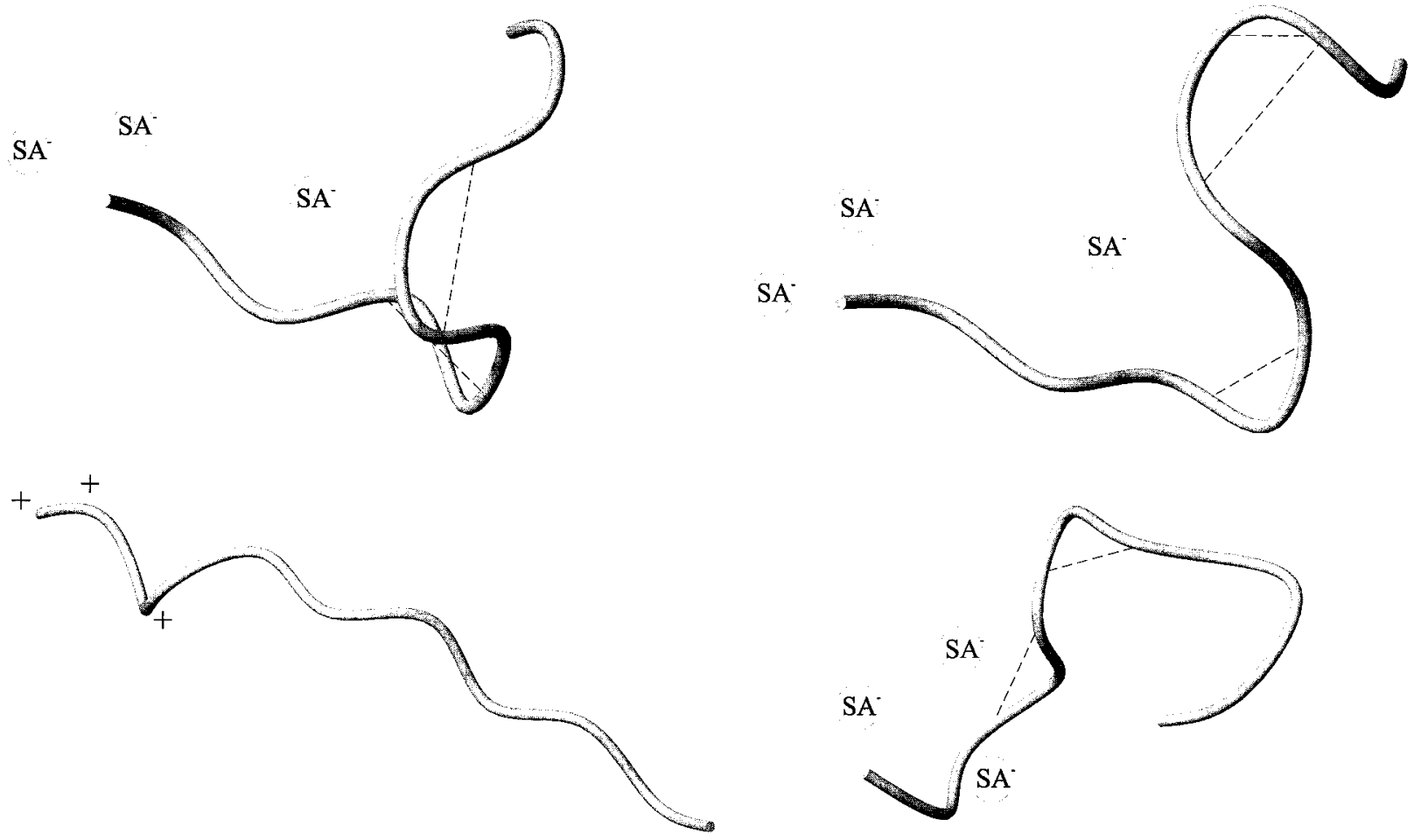

Figure 2. Typical backbone of the $\left[\mathrm{SP}^{3+}+3 \mathrm{SA}^{-}\right]$system (a, top left) on the surface, (b, top right) embedded into the SA matrix crystal, and (d, bottom right) in the gas phase. The three charges on the SP are neutralized by three $\mathrm{SA}^{-}$ions. (c, bottom left) Conformation of $\mathrm{SP}^{3+}$ in the gas phase.

molecules. A conformation similar to the previous embedded systems was observed for this configuration. The end-to-end distances of the buried SP species were comparable in the three systems $(\sim 18.6 \AA)$. Once embedded, the guest species maintained their conformation throughout the crystal growth. In the three embedded systems, the $\left[\mathrm{SP}^{3+}+3 \mathrm{SA}^{-}\right]$species were placed between the layers parallel to the (103) plane of the SA crystal. If the hydrophobic interactions between the (103) plane of the SA crystal and the guest species affect the guest conformation, it is reasonable to expect such a similarity. ${ }^{18}$ The guest species on the free surface of the crystal, however, is exposed to two different environments-vacuum on one side and crystal surface on the other-and, thus, a different conformation is attained.

The surface and embedded structures were also substantially different from the conformation of $\mathrm{SP}^{3+}$ in the gas phase (Figure 2c). In a vacuum, the $\mathrm{SP}^{3+}$ species exhibited an extended structure with random coils and no hydrogen bonds on the backbone. The average end-to-end distance of this extended $\mathrm{SP}^{3+}$ was $26 \AA$. This unfolding could be attributed to the three positive charges on the molecule. According to a recent study based on MD simulations, highly charged states of ionized lysozyme molecules undergo significant unfolding and lose their tertiary structure. ${ }^{44}$

However, the $\mathrm{SP}^{3+}$ ion in the gas-phase $\left[\mathrm{SP}^{3+}+3 \mathrm{SA}^{-}\right]$ complex exhibited a different conformation; two hydrogen bonds between Lys(3)-Gln(5) and $\mathrm{Gln}(6)$-Phe(7) and three turns on the backbone were observed in this structure (Figure 2d). In the $\left[\mathrm{SP}^{3+}+3 \mathrm{SA}^{-}\right]$complex, the charges on the $\mathrm{SP}^{3+}$ were neutralized by the negative charges of the $\mathrm{SA}^{-}$ions, therefore, allowed the complex to assume a much more compact structure with an end-to-end distance of $8.6 \AA$. This value was noticeably lower than the $12.1,18.6$, and $26 \AA$ end-to-end distances of the SP backbone in the $\left[\mathrm{SP}^{3+}+3 \mathrm{SA}^{-}\right]$complex placed on the surface, inside the host crystal, and the $\mathrm{SP}^{3+}$ in the gas phase, respectively.
Since in larger guest molecules the ionizable sites can be buried inside the structure, the conformation of the guest species within the matrix crystal may influence the accessibility of these sites for ionization. In a recent study, experimental evidence showed that the secondary structure of the investigated peptides had a direct effect on the ion formation in MALDI. ${ }^{3}$ Peptides with disturbed secondary structures showed higher signal intensities than those with stable secondary structures $(\alpha$-helix and $\beta$-sheets). Although in this experiment it was not clear how the conformation of the guest species changed from solution during the embedding and desorption, it did seem to affect the ionization process in MALDI. The calculations presented above show that there is a significant difference between the conformations of the gas-phase and embedded species.

Desorption Conformations of the Guest Species. Laser excitation simulations were carried out on the four different equilibrated systems with the $\left[\mathrm{SP}^{3+}+3 \mathrm{SA}^{-}\right]$species on the surface and buried at the third, sixth, and ninth layers. Laser irradiation in the systems was represented by an increase in the temperature of the heat bath coupled to the matrix molecules. Using the Nose-Hoover method, the rate of energy flow from the bath to the system was adjusted according to the variation of laser intensity during a pulse, resulting in a rapid temperature rise of the matrix crystal and evaporation of the system. Due to the strong absorption of the matrix molecules and the negligible absorption of the guest species at the laser wavelength, (e.g., $337 \mathrm{~nm}$ for the nitrogen laser), the energy transferred to the guest species was mostly the result of their interaction with the matrix molecules.

The conformational changes of the guest species were monitored as the heating of the system proceeded. We observed that following desorption the guest species had a significantly different conformation from the one they had on the surface or inside the matrix crystal. Figure 3 shows the desorption conformations obtained for the guest species that has originated from (a) the surface, (b) the third layer, and (c) the sixth layer 


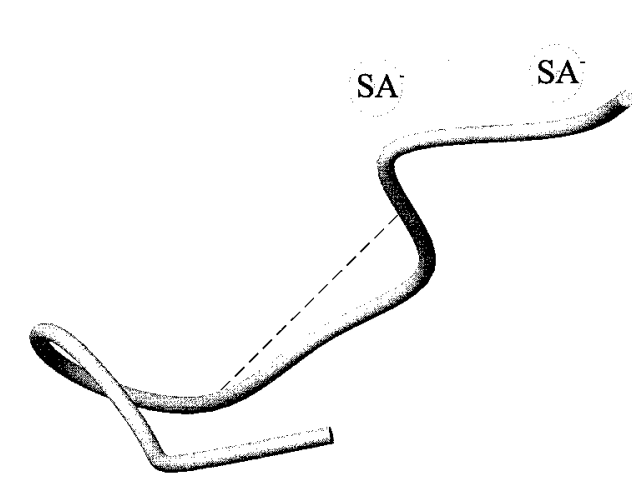

$\mathrm{SA}$
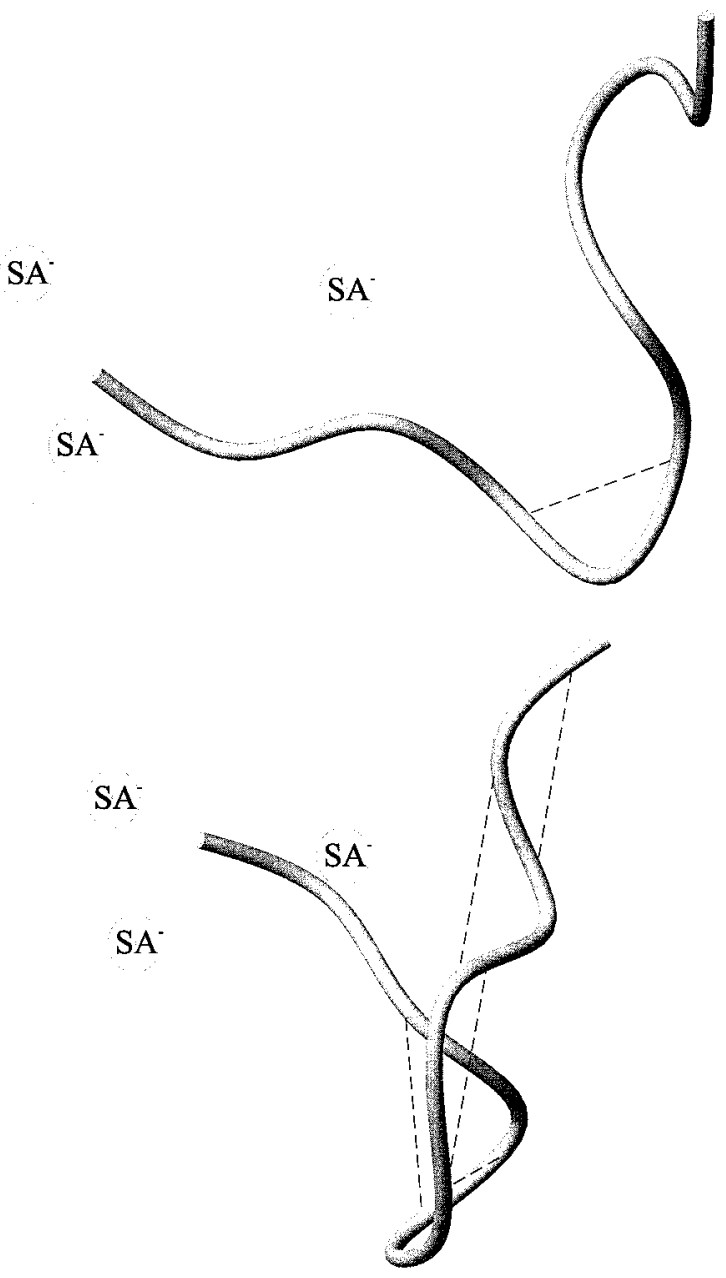

Figure 3. Gas-phase conformations of the guest species after desorption from (a, top) the surface, (b, middle) the third layer, and (c, bottom) the sixth layer.

of the crystal. Due to the reduced restriction on the $\left[\mathrm{SP}^{3+}+\right.$ $\left.3 \mathrm{SA}^{-}\right]$species in the gas phase and to the elevated temperature, no stable conformations were observed.

Figure $3 \mathrm{a}$ presents a particular conformation of the guest species desorbed from the surface of the crystal at $\sim 70$ ps after the start of the simulation. A single hydrogen bond between Pro(4) and Gln(6) and a random coil were observed in this structure. Figure $3 b$ indicates the backbone of SP ejected from the third layer at $\sim 90 \mathrm{ps}$ into the simulation. The hydrogen bond between Pro(4) and Gln(6) was also observed in this structure; however, an additional turn appeared in the conformation at Phe(8). The gas-phase structure of the guest species at $\sim 90 \mathrm{ps}$ released from the sixth layer is shown in Figure 3c. Up to five hydrogen bonds were observed on the backbone of the peptide with only one turn. The guest species originating from the ninth layer did not leave the crystal within the 100 ps duration of the simulations. Studying the conformation of SP at various times of the desorption showed that no preferred conformation existed for the guest species upon release from the crystal.

Throughout and after desorption, the three $\mathrm{SA}^{-}$ions kept their position relative to the $\mathrm{SP}$ species; i.e., the $\left[\mathrm{SP}^{3+}+3 \mathrm{SA}^{-}\right]$ complex was preserved. The electrostatic interaction between the $\mathrm{SP}^{3+}$ and the $\mathrm{SA}^{-}$ions was sufficient to keep the complex intact in the energetic environment created by the laser irradiation. The complex appeared in the gas phase rationalizing how, for example, matrix - analyte adduct peaks appear in MALDI mass spectra. After protonated and alkalinated molecular ion peaks, matrix - analyte adducts are among the most commonly observed peaks in MALDI spectra. This observation may also explain how certain noncovalent complexes (e.g., ion pairs) survive volatilization in MALDI.

In an effort to provide a simple measure of how stretched the structures were, we investigated the radius-of-gyration, $R_{\mathrm{gyr}}$, of the guest species, before and throughout the desorption process. The $R_{\mathrm{gyr}}$ is defined as the root-mean-square distance between the end and the center of gravity of a polymer chain. It is considered a measure of the compactness of a polymer molecule. ${ }^{45}$ Figure 4 shows the radius-of-gyration for the three desorbed peptides discussed above. At $300 \mathrm{~K}$, adsorbed on the surface of the crystal, the guest species had an $R_{\text {gyr }}$ of $\sim 6.5 \AA$ (Figure 4a). After $50 \mathrm{ps,}$, when the $\left[\mathrm{SP}^{3+}+3 \mathrm{SA}^{-}\right]$species had escaped from the surface of the crystal, the fluctuations in the $R_{\text {gyr }}$ increased. Similar trends were observed for the SP species embedded in the crystal. The $R_{\text {gyr }}$ value for guest species embedded inside the crystal was slightly higher than that on the surface, $7.0 \AA$ for the third layer and $7.25 \AA$ for the sixth layer compared to the $6.5 \AA$ surface value (Figure $4 b, c)$. Heating led to desorption, and the guest species became unstructured in all systems. It can be observed in Figure $4 \mathrm{~b}$ that while the $\left[\mathrm{SP}^{3+}\right.$ $+3 \mathrm{SA}^{-}$] remains embedded in the crystal (before phase transition), the $R_{\mathrm{gyr}}$ slightly increases with increasing temperature. Large fluctuations in the $R_{\mathrm{gyr}}$, however, are only observed after the release of the guest species into the gas phase. Figure 4c shows $R_{\mathrm{gyr}}$ of the guest species released from the sixth layer. In this system, the $R_{\text {gyr }}$ fluctuated around a constant value $(\sim 7.25$ $\AA$ ) while embedded under six layers of SA molecules. At 80 ps, past the phase transition, larger fluctuations appeared in the $R_{\text {gyr. }}$. These large variations also confirmed that no preferred conformation existed for the guest species in the gas phase.

Host Crystal Phase Transition. The dynamics of energy transfer between the guest and host subsystems in MALDI has been the target of a number of investigations. ${ }^{29,30,34,35}$ These studies rationalized the absence of guest decomposition in MALDI as a consequence of inefficient energy transfer in a nonequilibrium system. To further explore the energy transfer during the desorption process; we investigated the temperature histories of the guest and matrix molecules in the system. For this purpose, kinetic and internal temperatures of the SP species were compared with the kinetic temperatures of the SA molecules.

Figure 5a shows the guest and matrix temperature profiles for the SP-SA system with the guest species buried in the third layer. Both the kinetic and the internal temperatures of the guest species were lower than the temperature of the matrix molecules throughout the desorption process. However, the observed temperature differences varied throughout the simulation. Three stages were distinguished during the first 100 ps. From the initiation of the heating process until the escape of the guest 

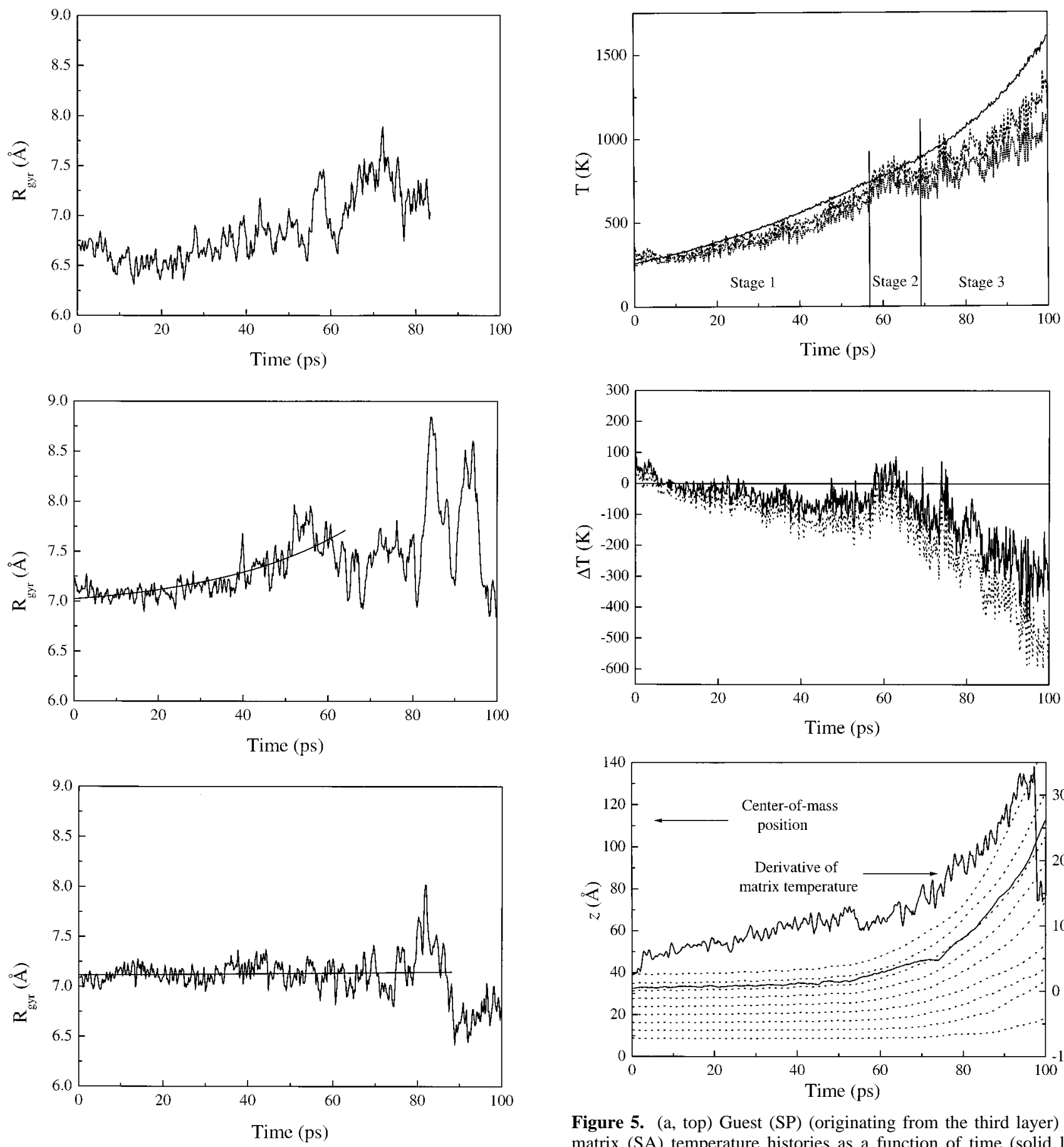

Figure 4. Changes in the radius-of-gyration of the $\mathrm{SP}^{3+}$ species during the desorption simulations. The initial position of the guest was (a, top) the crystal surface, (b, middle) the third layer, and (c, bottom) the sixth layer.

species from the crystal, the temperature of the matrix and guest species stayed close. During this stage (stage 1), due to the close interaction of the guest and matrix molecules within the crystal, the calculated temperatures followed a similar trend. At the start of desorption ( $\sim 60 \mathrm{ps})$, the kinetic temperature of the guest species became equal to the matrix temperature. During the next stage (stage 2), the guest species resided in a dense plume of the matrix molecules; therefore, there was efficient energy exchange. For the same reason, this stage could also be viewed as conducive to cluster mediated processes, possibly leading to ionization. Upon desorption and as a consequence of the expansion of the matrix plume, the interaction between the matrix and guest species decreased. Because of this decoupling,

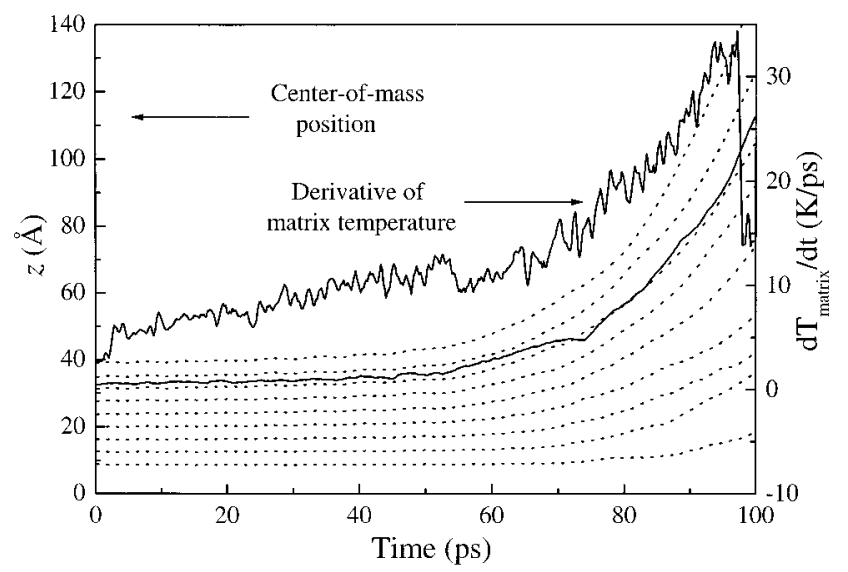

Figure 5. (a, top) Guest (SP) (originating from the third layer) and matrix (SA) temperature histories as a function of time (solid line, matrix kinetic temperature; dashed line, guest kinetic temperature; and dotted line, guest internal temperature). (b, middle) Difference between the kinetic temperatures of the matrix and guest species (solid line) and the internal temperature of the guest and the kinetic temperature of the matrix (dashed). (c, bottom) Center-of-mass position histories for the guest (solid line, left axis) and for individual layers of matrix molecules (dashed line, left axis) and the derivative of matrix temperature (solid line, right axis) during a 100 ps simulation.

a divergence between the matrix and guest temperatures developed (stage 3 in Figure 5a). These observations were also true for the other three investigated systems in this study. The consistently and increasingly lower internal energy of the guest species during stage 3 can account for the intact desorption or significantly limited fragmentation of large molecules in MALDI. Figure $5 \mathrm{~b}$ accentuates this effect by plotting temperature differences. The solid line indicates the difference between the kinetic temperature of the guest and that of the matrix, 
whereas the dotted line shows the difference between the internal temperature of the guest species and the kinetic temperature of the matrix particles. It was consistently observed that the internal temperature of the guest species lagged substantially more behind than its kinetic temperature. This finding was further evidence for the presence of an energy-transfer bottleneck in the system.

In Figure $5 \mathrm{c}$ the center-of-mass positions of the guest and matrix species are shown together with the derivative of the matrix temperature. Starting at $\sim 60 \mathrm{ps}$ (stage 2 ), the top layers of the matrix and the guest species departed from the crystal. At this time, a pronounced increase in the slope of the temperature derivative curve was also observed. We suggest that this change during stage 2 has corresponded to a phase transition in the matrix lattice. Coincidentally, the desorption process was initiated and the guest was released from the crystal structure. It is interesting to note that during this phase transition, the temperatures of guest and matrix molecules have converged. This effect was the result of the breakdown of the crystal structure that led to greater interaction between the matrix and guest species. The intimate interaction and similar temperatures of guest and matrix particles during stage 2 point to the importance of low sublimation temperature in MALDI. In matrices with high sublimation points the release of the guest molecules is delayed, leading to further energy transfer and potentially increased fragmentation.

Following the center of mass of the individual matrix layers revealed that the matrix material was removed layer by layer. This "peeling" type mechanism is generally associated with thermal desorption. Other mechanisms (phase explosion, spallation, etc.) can also be observed at higher heating rates (higher irradiances) but are not discussed in this contribution.

Desorption Velocities. Investigation of the different systems showed that, depending on the burial depth, the ejection of the guest species occurred at different times and velocities. In the first simulation, the $\left[\mathrm{SP}^{3+}+3 \mathrm{SA}^{-}\right]$species escaped from the surface of the crystal at $\sim 50 \mathrm{ps,} \mathrm{while} \mathrm{in} \mathrm{the} \mathrm{second} \mathrm{simulation}$ with the guest starting from the third layer the release occurred at $\sim 64 \mathrm{ps}$. The velocities observed for these two systems were relatively close, 176 and $167 \mathrm{~m} / \mathrm{s}$ for the first and second cases, respectively. The $\left[\mathrm{SP}^{3+}+3 \mathrm{SA}^{-}\right]$system, buried in the sixth layer of the SA crystal entered the gas phase at $\sim 85$ ps into the simulation with a liftoff velocity of $108 \mathrm{~m} / \mathrm{s}$. Consistently lower velocities and increasing time delays were observed for molecules embedded deeper inside the crystal. For example, the $\left[\mathrm{SP}^{3+}+3 \mathrm{SA}^{-}\right]$system implanted into the ninth layer of the crystal did not even leave the crystal within the 100 ps simulation time.

Thus, it is expected that the width of the velocity and departure time distributions depends on the thickness of the layer removed by the laser pulse. There are two ways to limit the thickness of this layer: to work at close to threshold irradiance and to utilize thin layers of the sample. Correspondingly, improved spectrum quality (e.g., higher resolution) can be observed at near-threshold irradiances and in the case of sample preparation techniques that lead to the formation of very thin samples. ${ }^{46}$

In summary, we have gained insight into various aspects of matrix-guest co-deposition and co-desorption in MALDI. During the embedding process, changes in the guest species conformation were investigated. While the guest species placed on the surface of the host crystal exhibited two hydrogen bonds on the backbone and one turn, the conformation of the embedded species contained three hydrogen bonds and two turns. Inde- pendent of the burial depth, the conformation of the guest species within the crystal remained the same. After laser heating and during the desorption process, no preferred stable gas-phase conformation was observed. Liftoff velocities of the guest species embedded in various layers of the host crystal and on the surface were similar to the liftoff velocities of the matrix molecules around them. This is an indication of the entrainment of guest species by the matrix plume. Investigation of the energy histories of the matrix and guest species showed lower internal and kinetic temperatures for the latter, which could explain the absence of fragmentation in MALDI. Results also showed that maximum energy transfer occurred during the phase transition of the host crystal at which time the guest species were released from the crystal. Examining the noncovalent complex of the guest species and sinapinic acid anions showed that the complex remained intact throughout the desorption process. A similar phenomenon is often reflected in MALDI mass spectra, in the form of guest-matrix adduct peaks.

Acknowledgment. The authors acknowledge the financial support of the National Science Foundation (Grant No. CHE9873610). We would also like to thank Dr. A. Bencsura for the helpful discussions, Dr. S. Kristyan for his constructive remarks on the manuscript, and J.M. Gauntt for writing a code used in part of the data analysis.

\section{References and Notes}

(1) Nelson, R. W.; Dogruel, D.; Williams, P. Rapid Commun. Mass Spectrom. 1995, 6, 625 . 260.

(2) Berkenkamp, S.; Kirpekar, F.; Hillenkamp, F. Science 1998, 281,

(3) Wenschuh, H.; Halada, P.; Lamer, S.; Jungblut, P.; Krause, E. Rapid Commun. Mass Spectrom. 1998, 12, 115.

(4) Figueroa, I. D.; Russell, D. H. J. Am. Soc. Mass Spectrom. 1999 10,719

(5) Laiko, V. V.; Baldwin, M. A.; Burlingame, A. L. Anal. Chem. 1999, 72,652 .

(6) Tang, X.; Callahan, J. H.; Zhou, P.; Vertes, A. Anal. Chem. 1995, 67,4542 .

(7) Gidden, J.; Wyttenbach, T.; Batka, J. J.; Weis, P.; Jackson, A. T.; Scrivens, J. H.; Bowers, M. T. J. Am. Chem. Soc. 1999, 121, 1421.

(8) Gidden, J.; Jackson, A. T.; Scrivens, J. H.; Bowers, M. T. Int. J. Mass Spectrom. Ion Processes 1999, 188, 121.

(9) Gidden, J.; Wyttenbach, T.; Jackson, A. T.; Scrivens, J. H.; Bowers, M. T. J. Am. Chem. Soc. 2000, 122, 4692

(10) Callahan, J. H.; Galicia, M. C.; Vertes, A. Proceedings of the 48th ASMS Conference on Mass Spectrometry and Allied Topics, Long Beach, CA, 2000; ASMS: Santa Fe, NM, p 210.

(11) Williams, R. W.; Weaver, J. L. J. Biol. Chem. 1990, 265, 2505.

(12) Chassaing, G.; Convert, O.; Lavielle, S. Eur. J. Biochem. 1986 $154,77$.

(13) Schwyzer, R.; Rolka, K.; Krzystof, R. Helv. Chim. Acta 1986, 69, 1789 .

(14) Erne, D.; Rolka, K.; Schwyzer, R. Helv. Chim. Acta 1986, 69, 1807. 2123 .

(16) Wymore, T.; Wong, T. C. Biophys. J. 1999, 76, 1199.

(17) Gill, A. C.; Jennings, K. R.; Wyttenbach, T.; Bowers, M. T. Int. J. Mass Spectrom. Ion Processes 2000, 195/196, 685.

(18) Beavis, R. C.; Bridson, J. N. J. Phys. D 1993, 26, 442.

(19) Strupat, K.; Kampmeier, J.; Horneffer, V. Int. J. Mass Spectrom. Ion Processes 1997, 169/170, 43.

(20) Horneffer, V.; Dreisewerd, K.; Ludemann, H.-C.; Hillenkamp, F.; Lage, M.; Strupat, K. Int. J. Mass Spectrom. Ion Processes 1999, 185187,859 .

(21) Juhasz, P.; Biemann, K. Proc. Natl. Acad. Sci. U.S.A. 1994, 91 4333.

(22) Woods, A. S.; Buchsbaum, J. C.; Worrall, T. A.; Berg, J. M.; Cotter, R. J. Anal. Chem. 1995, 67, 4462.

(23) Tang, X.; Sadeghi, M.; Olumee, Z.; Vertes, A. Rapid Comm. Mass. Spectrom. 1997, 11, 484.

(24) Knochenmuss, R.; Vertes, A. J. Phys. Chem. B 2000, 104, 5406

(25) Bencsura, A.; Vertes, A. Chem. Phys. Lett. 1995, 247, 142.

(26) Bencsura, A.; Navale, V.; Sadeghi, M.; Vertes, A. Rapid Commun. Mass Spectrom. 1997, 11, 679. 
(27) Zhigilei, L. V.; Kodali, P. B. S.; Garrison, B. J. J. Phys. Chem. B 1997, 101, 2028.

(28) Zhigilei, L. V.; Kodali, P. B. S.; Garrison, B. J. J. Phys. Chem. B 1998, 102, 2845.

(29) Wu, X.; Sadeghi, M.; Vertes, A. J. Phys. Chem. B 1998, 102, 4770.

(30) Dutkiewicz, L.; Johnson, R. E.; Vertes, A.; Pedrys, R. J. Phys. Chem. A 1999, 103, 2925.

(31) Zhigilei, L. V.; Garrison, B. J. Appl. Phys. Lett. 1999, 74, 1341.

(32) Zhigilei, L. V.; Garrison, B. J. Appl. Phys. A 1999, 69, S75.

(33) Kodali, P. B. S.; Zhigilei, L. V.; Garrison, B. J. Nucl. Instrum. Methods Phys. Res., Sect. B 1999, 153, 167.

(34) Vertes, A.; Gijbels, R.; Levine, R. D. Rapid Commun. Mass Spectrom. 1990, 4, 228

(35) Vertes, A.; Levine, R. D. Chem. Phys. Lett. 1990, 171, 284.

(36) Kinsel, G. R.; Preston, L. M.; Russell, D. H. Biol. Mass Spectrom. 1994, 23, 205.

(37) Brooks, B. R.; Bruccoleri, R. E.; Olafson, B. D.; Swaminathan, S.; Karplus, M. J. Comput. Chem. 1983, 4, 187.
(38) Schmidt, M. W.; Baldridge, K. K.; Boatz, J. A.; Elbert, S. T.; Gordon, M. S.; Jensen, J. H.; Koseki, S.; Matsunaga, N.; Nguyen, K. A. Su, S. J.; Windus, T. L.; Dupuis, M.; Montgomery, J. A. J. Comput. Chem. 1993, 14, 1347.

(39) Nose, S. J. Chem. Phys. 1984, 81, 511.

(40) Hoover, W. G. Phys. Rev. A 1985, 31, 1695.

(41) Laaksonen, L. J. Mol. Graphics 1992, 10, 33.

(42) Sayle, R. RasMol V2.6, Molecular Visualization Program, Glaxo Wellcome Research and Development; Glaxo Wellcome: Stevenage, Hertfordshire, U.K., 1992.

(43) Koradi, R.; Billeter, M.; Wüthrich, K. J. Mol. Graphics 1996, 14 51

(44) Reimann, C. T.; Velázquez, I.; Tapia, O. J. Phys. Chem. B 1998 102,9344

(45) Carraher, C. E., Jr.; Seymour, R. B. Seymour/Carraher's Polymer Chemistry: An Introduction, 4th ed.; Dekker: New York, 1996; p 31.

(46) Sadeghi, M.; Vertes, A. Appl. Surf. Sci. 1998, 127-129, 226. 